\title{
BMP Evaluation for Nutrient Control in a Subtropical Reservoir Watershed Using SWAT Model
}

\author{
Tzyy-Woei Chu, Assistant professor \\ Yu-Cheng Lin, Graduate student \\ Dept. of Civil Engineering \\ National Taipei University of Technology \\ Taipei, Taiwan \\ twchu@ntut.edu.tw
}

\author{
Yu-Chi Huang, Ph.D. student \\ Dept. of Environmental Engineering \\ National Cheng Kung University \\ Tainan, Taiwan \\ e_7_@hotmail.com
}

\author{
Adel Shirmohammadi, Professor \\ Assoc.Dean for Research and Assoc. Director of MAES \\ University of Maryland \\ College Park, USA \\ ashirmo@umd.edu
}

\begin{abstract}
The eutrophication problem that results from the inappropriate management in upstream watershed is prevailing among most reservoirs in Taiwan. As a result, implementation of appropriate Best Management Practices (BMPs) in upland watershed has proved to be the most effective measure to reduce or alleviate the nutrient loadings and the risk of eutrophication. This study aims to employ the uncertainty analysis and risk probability on SWAT (Soil and Water Assessment Tool) simulation for assessing the effectiveness of various BMP scenarios implemented in Feitsui reservoir watershed. The SWAT model was applied to assess the water quality impacts due to the implementation of various BMP scenarios. Five BMPs were selected and implemented on each agricultural land for overall evaluation of pollution reduction efficiency both at field and watershed scales. Finally, the effects of uncertainty in model input parameters on pollution reduction efficiency of BMPs are evaluated using constrained Monte Carlo simulation based on Latin hypercube sampling (LHS) and Orthogonal sampling (OS) schemes. Moreover, the Cumulative Distribution Functions (CDF) of average annual TP and chlorophyll a concentrations were created for further assessment on eutrophication risk. The simulation results show that the reduction of eutrophication risk approaches $13 \%$ (from 15 to $2 \%$ ) for Caelson Trophic Satus Index (CTSI) methods based on implementation of 5 BMPs (a combined BMP scenario). Overall, this study concludes that incooperating uncertainty analysis in modeling will provide model output with specific probability, which not only assists the decision-maker in developing BMP and TMDL plans but also assesses the reliability of NPS pollution control strategies and risk of water quality degradation.
\end{abstract}

Index Terms - Best Management Practices (BMPs), nonpoint source pollution, nutrient, SWAT model, uncertainty analysis.

\section{INTRODUCTION}

Reservoirs have been widely built to manage the water supplies in Taiwan. However, the eutrophication problem that results from the inappropriate managements in upstream watersheds is prevailing in most reservoirs. The Feitsui Reservoir has been providing raw water for the Greater Taipei
Metropolitan area as a long-term source of water supply for domestic purpose, and its water quality has been well protected by associated authorities. Nevertheless, recent monitoring data [1] and research results suggest that the water quality has reached the near-eutrophic state, especially when Taipei-Ilan expressway was constructed and came to operate. During the past decades, point source pollution has been reduced effectively through regulation that the Feitsui reservoir administration has enforced, and therefore, nonpoint source pollution is proved to be the major contribution to the eutrophication problem.

Best Management Practices (BMPs), both structural and non-structural, are implemented as either a single practice or as a system of practices to reduce nonpoint source pollution inputs to receiving water bodies. The effectiveness of BMPs at watershed level is difficult to identify individually due to the complex physiographic nature of watershed and the interactions between different pollutants, and meanwhile, the continuous water quality monitoring is expensive and spatially impractical in mixed land use watersheds; hence, watershed scale models have been recognized as effective tools to evaluate the long-term water quality impacts due to the implementation of different BMP scenarios in spatially diverse watersheds.

The accuracy of model predictions heavily relies on how precise the input parameters are. The values of input parameters used in general modeling are usually estimated due to the unavailability in the measured values. The risk of model error thus rises with the increasing degree of uncertainty associated with the inaccurate input parameters. Therefore, incorporating uncertainty analysis due to variability in model's input parameter values has gained increasing research importance in assessing model capabilities [2].

Instead of providing a single estimate, the stochastic methods can lead to more precise statement about the uncertainty of model results and thus enhancing the model utility and effectiveness [3]. Inasmuch as numerous researches 
have applied the stochastic modeling approaches that incorporated sensitivity with uncertainty analysis techniques, Shirmohammadi [2 and 4] concluded that stochastic approaches capture field heterogeneity better than a standard deterministic approach did. This study aims to employ the uncertainty analysis and risk probability on SWAT (Soil and Water Assessment Tool) simulations for assessing the effectiveness of various BMP scenarios implemented in Feitsui reservoir watershed. The SWAT model's performance was enhanced by means of generating the corresponding probability distributions of model outputs. In addition, the generated Cumulative Distribution Functions (CDF) of average annual TP and chlorophyll a concentrations were created for further assessment of eutrophication risk based on the Carlson Trophic Status Index (CTSI) method.

\section{METHODS AND MATERIALS}

\section{A. SWAT model Description}

SWAT is a complex, physically based model with spatially explicit parameterization. It has the ability to assess the impacts of different management practices on water supplies and pollutant loadings in watersheds and large river basins on a long-term basis. SWAT is also integrated into US-EPA's BASINS (Better Assessment Science Integrating Point and Nonpoint Sources) framework. It is a continuous time model that operates on a daily time step to perform simulations up to one hundred years by using measured and/or stochastically generated weather data. The precipitation data required by SWAT may be read into the model in daily or sub-daily time increments. The smallest unit of simulation can be either a subwatershed or a grid cell. Each subwatershed is divided into several land use and soil combinations which are called Hydrologic Response Units (HRUs) based on threshold percentages that are user defined [5]. HRUs are defined by first selecting land uses whose percentages (based on area) are greater than the user-defined land use threshold percentage and within those selected land uses, by selecting the soils whose percentages are greater than user-defined soil threshold percentage [6]. Geographical information system (GIS) interface was also developed to reduce spatial data collection and manipulation time, and to allow the user to modify and analyze various alternative management practices efficiently. The major components of the SWAT model include hydrology, weather, sedimentation, soil temperature, crop growth, nutrients, pesticides, bacteria, agricultural management, channel routing, and reservoir routing.

\section{B. Uncertainty analysis}

The model simulations should be sufficiently numerous to reliably estimate the probability distribution of the output variables for applying Monte Carlo Simulation (MCS) analysis. However, this requires considerable computing efforts. The LHS method can be used to efficiently estimate uncertainty in the model output when the MCS analysis become prohibitive due to computing limitation. Iman and Helton [7] found that the LHS sample size $(n)$ could be obtained with $n>(4 / 3) \mathrm{P}$ (input variables) to achieve good results. This is a significant improvement relative to standard Monte Carlo techniques, which typically require at least an order of magnitude more runs per input variable [3].

In this study, the effects of variability and distribution in input parameter values on model output parameters are evaluated by employing constrained Monte Carlo simulation based on Latin hypercube Sampling (LHS) and Orthogonal Sampling (OS) scheme. The orthogonal sampling was added to achieve better equidistribution property than LHS alone. Considering a simple LHS scheme with two parameters $\left(\mathrm{P}_{1}\right.$ and $\mathrm{P}_{2}$ ) and four samples $(n=4)$ is depicted in figure 1. Figure 1 shows that the LHS scheme is not an equidistributed sampling scheme. Orthogonal sampling [8] divides the sample space into subspaces with equal probability. Each subspace is limited to the same sampling by LHS (e.g., each subspace has the same sampling points). Figure 2 indicates the sampling scheme involving combination of Orthogonal sampling and LHS. This combination will improve the sampling technique to more equidistributed sample space.

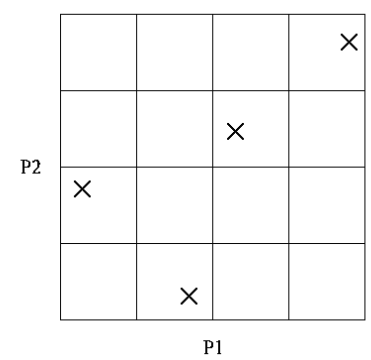

Fig. 1. Latin hypercube sampling scheme $(\mathrm{P}=2, n=4)$

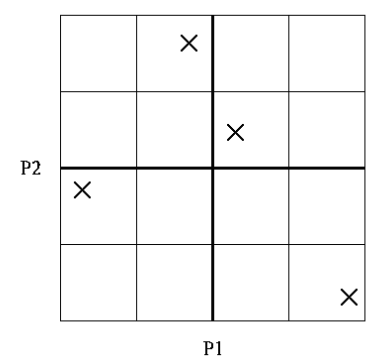

Fig. 2. Sampling scheme involving combination of Orthogonal sampling and LHS

\section{Risk assessment of reservoir eutrophication}

The generated probability distributions of TP (total phosphorus) and chlorophyll a concentrations from uncertainty analysis will be further applied to assess the risk of eutrophication in Feitsui reservoir. The risk will be estimated based on the classification of eutrophic status set by the Carlson Trophic Status Index (CTSI). Three factors including total phosphorous, chlorophyll a (CHA), and Secchi disk depth (SD) are adopted to establish the standards of eutrophic status (table 1). The average CTSI is calculated as in equation 1:

$$
\begin{aligned}
& C T S I=\frac{T S I(1)+T S I(2)+T S I(3)}{3} \\
& T S I(1)=60-14.41 \times \ln (\mathrm{SD}) \\
& T S I(2)=4.15+14.42 \times \ln (\mathrm{TP}) \\
& T S I(3)=30.6+9.81 \times \ln (\mathrm{CHA})
\end{aligned}
$$


TABLE I. TROPHIC STATE CLASSIFICATION DEFINED BY CTSI

\begin{tabular}{|c|c|}
\hline CTSI value & TROPHIC STATUS \\
\hline CTSI $<40$ & Oligotrophic \\
\hline $40 \leqq \mathrm{CTSI} \leqq 50$ & Mesotrophic \\
\hline CTSI $>50$ & Eutrophic \\
\hline
\end{tabular}

\section{BMP simulation and nutrient reduction assessment}

A variety of BMPs for nutrient reduction were evaluated, which range from streambank stabilization, terrace, field border (or filter strip), nutrient management, to crop residue management. Based on the function of the BMPs and hydrologic and water quality processes that are directly adjusted after BMPs implementation, the corresponding input parameters in SWAT were selected and altered as appropriate to represent each individual BMP.

\section{E. Description of Study Site}

The Feitsui Reservoir is located downstream of the Beishi stream and $20 \mathrm{~km}$ south of Taipei city. Land use in the selected $303 \mathrm{~km}^{2}$ Feitsui Reservoir watershed consists of a mixture of forest, tea farm, orchard, pasture, barren land, and residential area. The watershed is well protected from the excess development. More than $88 \%$ of watershed area is covered by forest. The main agricultural activity is tea farming $(3.86 \%)$ which is mostly distributed around the middle and upper portions of watershed (figure 3). During the past decades, point source has been reduced effectively through regulation that the Feitsui reservoir administration has enforced, and therefore, nonpoint source pollution is proved to be the major contribution to the eutrophication problem. The entire Feitsui Reservoir watershed was divided into 25 subwatersheds based on the stream distribution for the SWAT simulation. This allowed for consideration of significant spatial details. Twelve-year data (1998 2009) of weather, topography, hydrology, and water quality were collected for model calibration (1998 2003) and validation (2004 2009). The entire simulation contains two phases: watershed process and reservoir routing. Three statistical measures including efficiency coefficient $\left(\mathrm{E}_{\mathrm{N}-\mathrm{S}}\right)$, sorted efficiency coefficient $\left(\mathrm{E}_{\text {Sorted }}\right)$, and coefficient of determination $\left(\mathrm{R}^{2}\right)$ are used to evaluate the goodness-of-fit for model simulation.

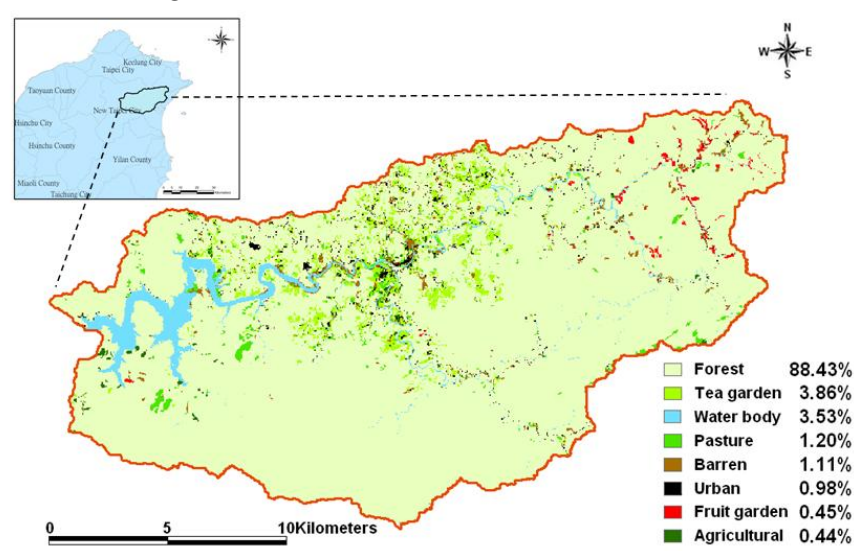

Fig. 3. The location and land use distribution of the Feitsui Reservoir watershed.

\section{RESULTS AND DISCUSSION}

\section{A. SWAT calibration and validation}

The result of sensitivity analysis for the 41 selected input parameters and the statistical results of the model performance for watershed and reservoir simulations during both calibration and validation periods are presented in Chu, et. al. [9]. The results of their study indicated that model predicted reasonably well for long-term simulation. It could be concluded that SWAT is a reasonable annual predictor of the watershed responses in assessing the impacts of different management systems on water supplies and nonpoint source pollution.

\section{B. BMP simulation}

The scenarios of five BMPs implemented individually or jointly on agricultural land were simulated by SWAT model. The average annual sediment, TP and TN (total nitrogen) reduction rates at watershed level were presented in table 2 . Table 2 indicates that only limited improvement of pollution reduction has been achieved when width of field border reaches over 10 meters. Consequently, field border (vegetated filter strip) of $10 \mathrm{~m}$ width was selected to perform the uncertainty analysis and it consist the 5 BMPs combination.

TABLE II. POLLUTION REDUCTION RATES OF VARIOUS BMP SCENARIOS AT WATERSHED LEVEL

\begin{tabular}{|l|c|c|c|}
\hline \multicolumn{1}{|c|}{ BMPs } & Sediment & TN & TP \\
\hline streambank stabilization & $42 \%$ & $2.02 \%$ & $6.04 \%$ \\
\hline terrace & $38 \%$ & $4.3 \%$ & $6.8 \%$ \\
\hline nutrient management (organic fertilizer) & $0.3 \%$ & $9.06 \%$ & $1.07 \%$ \\
\hline nutrient management (50\% fertilizer) & $0.3 \%$ & $10.1 \%$ & $2.04 \%$ \\
\hline field border (3m width) & $32.4 \%$ & $3.1 \%$ & $7.9 \%$ \\
\hline field border (5m width) & $39.8 \%$ & $4.7 \%$ & $9.7 \%$ \\
\hline field border (10m width) & $47.0 \%$ & $6.6 \%$ & $11.4 \%$ \\
\hline field border (15m width) & $54.3 \%$ & $8.7 \%$ & $12.9 \%$ \\
\hline field border (20m width) & $54.3 \%$ & $8.7 \%$ & $12.9 \%$ \\
\hline crop residue management & $1.6 \%$ & $0.4 \%$ & $0.78 \%$ \\
\hline 5 BMPs Combination & $72 \%$ & $22.3 \%$ & $26.7 \%$ \\
\hline
\end{tabular}

\section{Uncertainty analysis}

According to the results of previous work [9], the 19 input parameters sensitive to flow, sediment, and nutrient outputs were selected to perform uncertainty analysis. Each parameter was given an associated probability distribution based on data's characteristics, published literature [3], and professional judgment. The Latin hypercube sampling (LHS) scheme was performed to generate 100 samples, which were then incorporated to Monte Carlo simulation to produce 100 simulations. In addition, six highly sensitive parameters were sampled by LHS combining orthogonal sampling scheme to improve better equidistribution property than LHS alone.

The average TP concentrations in reservoir generated from 100 SWAT simulations was fitted to log-normal distribution 
via chi-square test and shown in figure 4 . In addition, the uncertainty effects on BMP efficiency of $\mathrm{TP}$ reduction at watershed level were compared in figure 5. Finally, the cumulative distribution of CTSI were established for pre-BMP and post-BMP, respectively, as displayed in figure 6 . The risk of eutrophication (CTSI > 50) was reduced from $15 \%$ to $2 \%$, which indicated an effective nutrient control via BMP implementation. Results presented in figures 5 and 6 clearly indicate that combined system of 5 BMPs is the most effective approach in reducing TP discharge from the study watershed. This is not to say that each individual BMP is not effective in their own, rather, it is showing that consideration of implanting combined BMPs is a better management strategy if both the local conditions and economics permit in using such strategy.

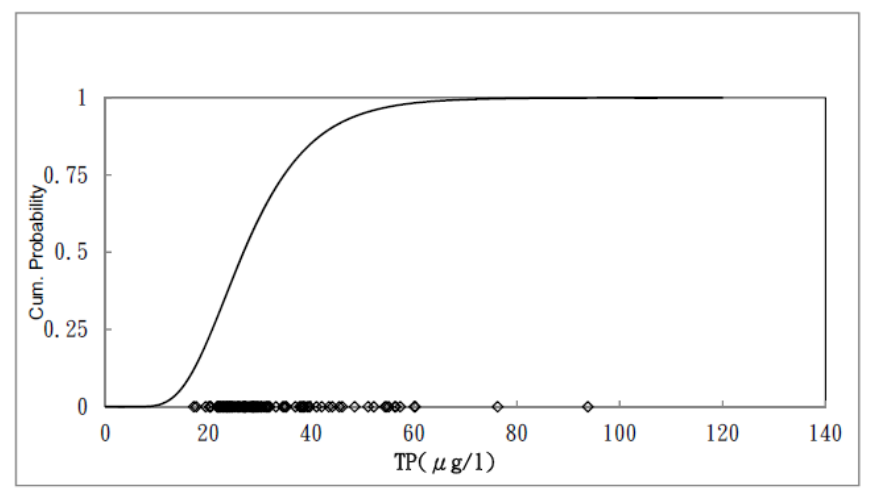

Fig. 4. The cumulative distribution of average TP concentration in reservoir obtained from 12-year simulation.

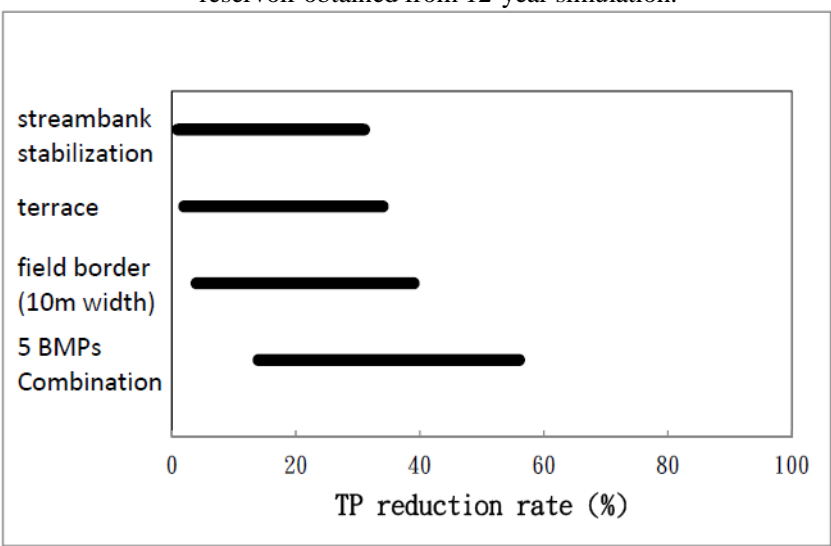

Fig. 5. The range of TP reduction rate at watershed level for various BMP scenarios.

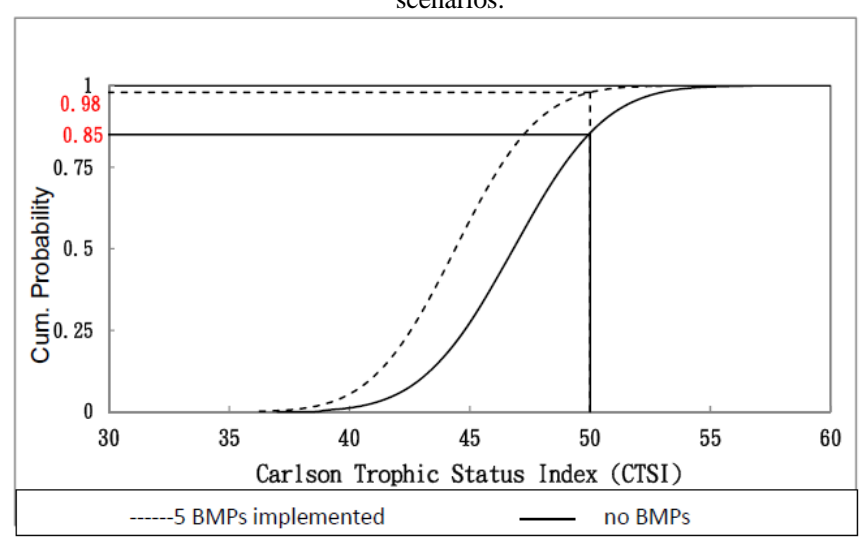

Fig. 6. The cumulative distribution of CTSI from 12-year simulation

\section{SUMMARY AND CONCLUSIONS}

Given the fact that the inherent uncertainty in model's predictions is a great concern for proper decisions for watershed management, a good ecosystem modeling practice should associate the uncertainty analysis with hydrologic/water quality models. This study's greatest benefit is to generate output values with their associated probability, which may have far-reaching influence on our decisions regarding the selection of proper management practices to improve water quality and preserve a healthy aquatic ecosystem. Moreover, these results can be further applied to assess the reliability or risk of any management practice for effective control of nonpoint source pollution. Overall, the uncertainty in mathematical models is a real issue and should be taken into consideration during the TMDL (Total Maximum Daily Load) development and BMP implementation.

\section{ACKNOWLEDGMENT}

Authors are grateful for funds provided by the department of engineering and applied sciences, National Science Council (NSC), Taiwan.

\section{REFERENCES}

[1] TFRA (Taipei Feitsui Reservoir Administration), Annual report of TFRA, Taipei, Taiwan, Republic of China (in Chinese), 2003.

[2] A. Shirmohammadi, I. Chaubey, R. D. Harmel, D. D. Bosch, R. Munoz-Carpena, C. Dharmasri, A. Sexton, M. Arabi, M. L. Wolfe, J. Frankenberger, C. Graff, and T. M. Sohrabi. Uncertainty in TMDL models. 2006, Trans. ASABE 49(4): 1033 -1049 .

[3] T.M. Sohrabi, A. Shirmohammadi, and H. Montas. Uncertainty in Nonpoint source pollution models and associated risks. 2002, Environmental Forensics, 3(2):179-189.

[4] A. Shirmohammadi, H. Montas, L. Bergstrom, K. Coyne, S. Wei, and T.J. Gish. Deterministic and stochastic prediction of atrazine transport in soils displaying macropore flow. Proceedings of the second international symposium on preferential flow, Honolulu, Hawaii, 2001, pp. 133-136.

[5] J.G. Arnold, R. Srinivasan, R.S. Muttiah, and J.R. Williams. Large area hydrologic modeling and assessment, part I: model development. 1998, Journal of the American Water Resources Association, 34(1):73-89.

[6] S.L. Neitsch, J.G. Arnold, J.R. Williams, J.R. Kiniry, and K.W. King. Soil and Water Assessment Tool (Version 2000) - Model Documentation. 2002, GSRWRL02-01, BREC 02-05, TR-191, Texas Water Research Institute, Texas A\&M University, College Station,TX. Available at: http://www.brc.tamus.edu/swat

[7] R. L. Iman, and J. C., Helton. A comparison of uncertainty and sensitivity analysis techniques for computer models. NUREG/CR-3904. Albuquerque, N.M.: Sandia National Laboratories, 1985.

[8] Z. Sándor, and P. András. Alternative sampling methods for estimating multivariate normal probabilities. 2004, Journal of Econometrics 120 (2): 207-234.

[9] T.W. Chu, Y. C. Chen, and Y. C. Huang. Nutrient Loading Assessment and Associated Uncertainty in a Subtropical Reservoir Watershed. 2010, ASAE Paper No. 1009861, ASAE, St. Joseph, MI 49085. 